\title{
COMPARAÇÃO ENTRE DOIS MÉTODOS DE AVALIAÇÃO DA VARIABILIDADE GENÉTICA EM VOLUME, DENSIDADE BÁSICA DA MADEIRA E MATÉRIA SECA DE Pinus tecunumanii (Schwd) Eguiluz e Perry
}

\section{A COMPARISON OF TWO METHODS OF ASSESSING GENETIC VARIABILITY IN VOLUME, WOOD DENSIY AND DRY MATTER OF Pinus tecunumanii (Schwd) Eguiluz and Perry}

\author{
Vicente Pongitory Gifoni Moura ${ }^{1}$ Ailton Teixeira do Vale ${ }^{2}$ Fabio Bakker Isaias $^{3}$
}

\section{RESUMO}

O volume, a densidade básica e o peso da matéria seca da madeira de oitenta e quatro árvores, de sete famílias de polinização aberta, procedência de Mountain Pine Ridge (MPR), Belize, de Pinus tecunumanii (Schwd) Eguiluz e Perry foram estimados aos 12 e 17 anos de idade em experimento instalado em Planaltina, Distrito Federal. Para todos os parâmetros avaliados, houve diferenças significativas entre famílias, exceto para densidade básica, medidas aos 17 anos de idade. Aos 12 anos de idade, foram testados dois métodos de avaliação para densidade básica da madeira; um com os três melhores indivíduos da parcela, e outro com todos indivíduos da parcela. As médias de densidade foram de $0,425 \mathrm{~g} / \mathrm{cm}^{3} \mathrm{e} 0,424 \mathrm{~g} / \mathrm{cm}^{3}$, praticamente iguais para as duas amostragens. A correlação da densidade básica entre as idades foi positiva e significativa, mostrando um ganho de $0,031 \mathrm{~g} / \mathrm{cm}^{3} \mathrm{em} 5$ anos. A herdabilidade da densidade básica calculada aos 12 anos de idade, em nível individual, para família e dentro de família, considerando-se a amostragem com três e seis indivíduos, foi de respectivamente $0,43,0,81,0,33$ e $0,47,0,63,0,37$. Como conclusão, as seleções para densidade básica devem ser feitas preferencialmente em idades quando a competição entre os indivíduos ainda não atingiu níveis elevados. Ganhos consideráveis podem ser obtidos na seleção de indivíduos ou famílias dentro da população de MPR, Belize, na região do Cerrado. Será necessário trabalhar com populações de maior base genética.

Palavras-chave: Pinus tecunumanii; densidade básica; matéria seca; herdabilidade.

\section{ABSTRACT}

Eighty-four trees, of seven open-pollinated families from the Mountain Pine Ridge (MPR), Belize provenance of Pinus tecunumanii (Schwd) Eguiluz e Perry were assessed for volume growth, wood density and dry matter at 12 and 17 years of age at Planaltina, Federal District. Family differences for all traits were significantly different except for wood density at 17 years of age. Two methods were used for wood density evaluation at 12 years of age; one with the three better individuals within each parcel, and another one with all individuals in each parcel. Average individual wood density were 0.424 and $0.425 \mathrm{~g} / \mathrm{cm}^{3}$, practically equal for the two samplings. There was a positive and significant wood density correlation between the two ages, with an increase of density of $0.031 \mathrm{~g} / \mathrm{cm}^{3}$ from the age 12 to 17 . Individual, family and within family heritability for wood density for the two kind of sampling ( 3 and 6 individuals), was of $0.43,0.81,0.33$ and $0.47,0.63,0.37$ respectively. Selections for wood density should be made preferably in ages when the competition between individuals still did not reach high levels. Sizable improvement both in volume and wood density can be made in the MPR, Belize population, in the Cerrado region. However, a larger genetic base is needed.

Key words: Pinus tecunumanii; wood density; dry matter; heritability.

\section{INTRODUÇÃO}

O gênero Pinus é o mais antigo da família Pinaceae, tendo surgido há aproximadamente 180 milhões de anos. Seu centro de origem é o Hemisfério Norte, ocorrendo entre latitudes de $0^{\circ}$ a $70^{\circ}$ e altitudes de 0 a

1. Engenheiro Florestal, Dr., Pesquisador da Embrapa Recursos Genéticos e Biotecnologia. Parque Estação Biológica, Final da Av. W5 Norte, CEP 70770-900, Brasília (DF).vmoura@ cenargen.embrapa.br

2. Engenheiro Florestal, Dr., Professor do Departamento de Engenharia Florestal, Faculdade de Tecnologia, Universidade de Brasília, Campus Universitário, CEP 70910-900, Brasília (DF). atvale@unb.br

3. Engenheiro Florestal, Administrador Parques Estaduais da Bahia, Centro de Recursos Ambientais, CRA. Av. J.J. Seabra, 403, CEP 47400-000, Xique-Xique (BA). fabio@cra.ba.gov.br

Recebido para publicação em 3/1/2002 e aceito em 26/11/2003. 


\section{$3.500 \mathrm{~m}$.}

Existem mais de cem espécies pertencentes a esse gênero e, entre elas, o Pinus tecunumanii (Schwd) Eguiluz e Perry que se destacou nas características de crescimento, forma do fuste e densidade da madeira em testes com espécies/procedência de Pinus tropicais, realizados em diversos países, inclusive na região dos Cerrados (Wright et al., 1986a; Wright, 1987; Wright et al., 1990; Wright, 1990; Birks e Barnes, 1990 e Moura e Santiago, 1991). Apesar dos bons resultados obtidos em crescimento, os plantios comerciais com essa espécie ainda são poucos, em razão da pequena oferta de sementes e ao pouco conhecimento de suas necessidades silviculturais, sobretudo em se tratando de plantios em grande escala (Moura et al., 1996).

O Pinus tecunumanii ocorre naturalmente entre as latitudes $13^{\circ}$ e $28^{\circ} \mathrm{N}$, nas montanhas da América Central e do sul do México. Essa espécie possui alto valor econômico, atingindo $55 \mathrm{~m}$ de altura com, pelo menos, $30 \mathrm{~m}$ de fuste livre de ramos e até $120 \mathrm{~cm}$ de diâmetro à altura do peito (DAP). O tronco apresenta forma cilíndrica e sua copa é compacta (Eguiluz-Piedra e Perry, 1983).

Dentre as características silviculturais, a altura, o diâmetro e a densidade básica da madeira são as mais importantes, pois estão relacionadas com a produtividade das florestas, orientando a elaboração e a condução de planos de cortes (Rosado, 1983).

A densidade básica está estreitamente ligada à qualidade da madeira, e é uma característica bastante utilizada por pesquisadores e melhoristas na determinação do uso final da madeira. Em espécies, procedências e progênies de Pinus, a densidade básica se tem mostrado bastante variável. Essa característica tem alta herdabilidade, superior às herdabilidades das características de crescimento. Nos estudos genéticos e de melhoramento florestal, as seleções normalmente são dirigidas para os aspectos de crescimento, relacionados a ganhos em volume e forma. Pouca atenção tem sido dada aos aspectos tecnológicos e de qualidade da madeira. Estes são considerados apenas nos processos de seleção secundária (Brasil et al., 1980).

Os objetivos do presente trabalho são: Comparar metodologias de amostragem quanto ao número de indivíduos a serem selecionados para a característica densidade básica da madeira; Avaliar os aspectos de crescimento, densidade básica da madeira e índice de matéria seca em famílias de polinização aberta de Pinus tecunumanii procedência de Mountain Pine Ridge, Belize, em duas idades, em área de Cerrado.

\section{MATERIAL E MÉTODO}

As sementes foram coletadas de sete árvores matrizes de Pinus tecunumanii, de Mountain Pine Ridge, Belize, a $17^{0} 00^{\prime} \mathrm{N}$ de latitude, e altitude variando de 610 a $680 \mathrm{~m}$ (Tabela 1).

TABELA 1: Altura, DAP (diâmetro a altura do peito) das árvores selecionadas e altitude de onde foram coletadas as sementes de Pinus tecunumanii.

TABLE 1: Seed source altitude and selected Pinus tecunumanii trees height, DBH (diameter at breast height).

\begin{tabular}{c|c|c|c}
\hline Árvore & Altura $(\mathrm{m})$ & DAP $(\mathrm{cm})$ & Altitude $(\mathrm{m})$ \\
\hline 444 & 16 & 28,5 & 660 \\
448 & 17 & 27,8 & 620 \\
452 & 16 & 24,5 & 620 \\
461 & 20 & 34,0 & 610 \\
462 & 19 & 25,3 & 660 \\
482 & 15 & 30,5 & 680 \\
487 & 18 & 26,5 & 660 \\
\hline
\end{tabular}

O material de Mountain Pine Ridge foi testado juntamente com outras procedências de Pinus oocarpa Schiede estabelecido pela Empresa Brasileira de Pesquisa Agropecuária (EMBRAPA) em Planaltina (latitude $15^{\circ} 35^{\prime} \mathrm{S}$, altura $1000 \mathrm{~m}$, e precipitação anual média de $1554 \mathrm{~mm}$ ), Distrito Federal, Brasil. O solo é um Latossolo Vermelho-Escuro, de baixa fertilidade natural e elevada acidez, com média a alta concentração de alumínio. A vegetação é caracterizada por Cerrado ralo com extrato herbáceo predominantemente de gramíneas. 
O plantio das mudas foi realizado em dezembro de 1983, com espaçamento de 3 x 3 m, em delineamento estatístico de blocos ao acaso balanceado com parcelas de seis árvores por família e com nove repetições. Todo experimento foi circundado com uma bordadura dupla de Pinus oocarpa.

O experimento foi avaliado aos 12 anos de idade (idade 1), quando os parâmetros de volume, densidade básica da madeira e matéria seca foram obtidos de todas as árvores da parcela (amostragem 1), e aos 17 anos de idade (idade 2) quando os parâmetros de volume, densidade básica da madeira e matéria seca foram obtidos apenas dos três melhores indivíduos na parcela (amostragem 2).

Em análises de crescimento, forma de fuste e diâmetro de ramos é comum que as mensurações sejam feitas em todos os indivíduos da parcela. Entretanto, quanto ao parâmetro densidade da madeira, a amostragem é feita apenas nas melhores árvores por parcela. Assim demonstram os trabalhos realizados por Gibson (1982), Wright (1986b), Lima et al. (1990) e Birks e Barnes (1990), nos quais em parcelas com até 25 plantas, apenas dois ou três indivíduos foram amostrados.

A redução do número de indivíduos amostrados, segundo Gibson (1982) tem como objetivo principal a diminuição de custo e de tempo e, conseqüentemente, a redução do volume de trabalho de campo e laboratorial. Além disso, deve-se levar em conta que, nos processos de seleção, árvores dominadas e defeituosas são descartadas nos processos de melhoramento, mesmo que estas tenham alta densidade. Além do mais, estudos têm demonstrado correlações fracas ou inexpressivas entre incremento volumétrico e densidade básica da madeira (Barrichelo et al., 1977 e Ferreira, 1970).

As amostras (baguetas) para determinação da densidade básica foram retiradas à altura do peito com o auxilio de Sonda Pressler de 5,5 mm de diâmetro interno, em toda extensão do diâmetro da árvore evitandose nós, seguindo sempre o sentido leste-oeste. As baguetas foram retiradas de três árvores fenotipicamente superiores (dominantes e co-dominantes) em quatro das nove repetições, num máximo de 12 amostras por família; o experimento é composto de sete famílias, totalizando 84 amostras, as quais foram consideradas nas análises.

Retiradas as baguetas, os ferimentos foram tratados com calda bordaleza para prevenção de infecções.

A determinação da densidade básica da madeira foi feita pelo método do máximo teor de umidade (Smith, 1954).

Os dados de densidade foram submetidos a uma análise de variância usando-se o SAS (Statistical Analysis Systems), procedimento GLM (General Linear Model). No modelo abaixo descrito, todos os componentes, exceto a média geral e as famílias, foram considerados aleatórios.

$$
Y_{i j k}=u+r_{i}+f_{j}+r f_{i j}+e_{i j k}
$$

Em que: $\mathrm{u}=$ média geral; $\mathrm{r}_{\mathrm{i}}=$ efeito de $\mathrm{i}$ blocos; $\mathrm{f}_{\mathrm{j}}=$ efeito de $\mathrm{j}$ famílias; $\mathrm{r}_{\mathrm{ij}}=$ erro em nível de médias de família; $\mathrm{e}_{\mathrm{ijk}}=$ variação de $\mathrm{k}$ árvores em i blocos e j famílias.

O volume da árvore sem casca foi calculado pela fórmula geral desenvolvida para árvores jovens por Ladrach (1986):

$$
\text { Volume }\left(\mathrm{m}^{3}\right)=0,00003 \mathrm{DAP}^{2} \mathrm{~h}
$$

Em que: $\mathrm{DAP}=$ diâmetro a altura do peito; $\mathrm{h}=$ altura.

Para cálculo da matéria seca, multiplicou-se o volume pela densidade básica da madeira de cada árvore.

Os componentes da variância foram estimados pelo método MIVQUEO, dentro do procedimento VARCOMP, SAS (1990). A herdabilidade em nível de indivíduos, de famílias e dentro de famílias foi calculada da maneira usual, exceto no que diz respeito ao coeficiente, em que se usou o valor de 0,33 , em vez de 0,25 . Admitiu-se um grau de endogamia de cerca de $10 \%$ nas populações pequenas nas quais as sementes de Pinus tecunumanii foram coletadas (Vasquez e Dvorak, 1996). Aumentando-se o coeficiente de 0,25 para 0,33 , os valores estimados da herdabilidade se tornam mais conservadores (Squillace, 1974). As expressões para estimativas das herdabilidades estão descritas abaixo: 
$\mathrm{h}_{\mathrm{i}}^{2}=$ herdabilidade individual

$\mathrm{h}_{\mathrm{f}}{ }^{2}=$ herdabilidade de família

$\mathrm{h}_{\mathrm{w}}{ }^{2}=$ herdabilidade dentro de família

Em que: $\mathrm{h}_{\mathrm{i}}{ }^{2}=\left(3 * \mathrm{~S}_{\mathrm{f}}{ }_{\mathrm{f}}\right) /\left(\mathrm{S}_{\mathrm{f}}{ }_{\mathrm{f}}+\mathrm{S}_{\mathrm{rf}}{ }_{\mathrm{rf}}+\mathrm{S}^{2}\right) ; \mathrm{h}_{\mathrm{f}}{ }^{2}=\left(\mathrm{S}_{\mathrm{f}}{ }_{\mathrm{f}}\right) /\left(\mathrm{S}_{\mathrm{f}}{ }_{\mathrm{f}}+\mathrm{S}_{\mathrm{rf}}{ }_{\mathrm{rf}} / \mathrm{r}+\mathrm{S}^{2} / \mathrm{nr}\right) ; \mathrm{h}_{\mathrm{w}}{ }^{2}=\left(2 * \mathrm{~S}_{\mathrm{f}}{ }_{\mathrm{f}}\right) /\left(\mathrm{S}_{\mathrm{rf}}{ }_{\mathrm{rf}}+\mathrm{S}^{2}\right)$.

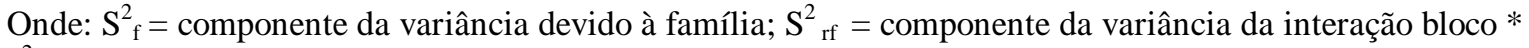
família; $S^{2}=$ componente da variância do erro; $\mathrm{n}=$ número de árvores por parcela e $\mathrm{r}$ é número de blocos.

\section{RESULTADOS E DISCUSSÃO}

Aos 12 anos de idade a média das densidades básicas entre as avaliações realizadas, com três ou seis indivíduos por parcela, foi de respectivamente 0,425 e $0,424 \mathrm{~g} / \mathrm{cm}^{3}$ (Tabela 2). Não houve diferença entre essas médias, mostrando que não faz nenhuma diferença se a densidade é determinada com parte ou com o total de indivíduos da parcela. Esses resultados mostram que a seleção de indivíduos com maior crescimento e/ou melhor forma (Gibson, 1982; Wright, 1990; Lima et al. 1990 e Birks e Barnes 1990), não acarreta diferenças significativas na média da densidade calculada. Houve ausência de correlação entre crescimento em volume e a densidade básica da madeira, também verificado por outros pesquisadores, em que as correlações entre volume e densidade básica da madeira foram fracas, inexpressivas ou mesmo inexistentes (Barrichelo et al., 1977 e Ferreira, 1970).

TABELA 2: Teste de Waller-Duncan para as características de densidade básica $\left(\mathrm{g} / \mathrm{cm}^{3}\right)$, volume $\left(\mathrm{m}^{3}\right)$ e índice de matéria seca $(\mathrm{kg})$, de famílias da procedência de Mountain Pine Ridge de Pinus tecunumanii, coletados em Planaltina, DF, aos 12 anos de idade: comparação entre médias amostrando-se todos os indivíduos da parcela e apenas os três indivíduos superiores (3 indiv.).

TABLE 2: Waller-Duncan test for the characteristics of wood density $\left(\mathrm{g} / \mathrm{cm}^{3}\right)$, volume $\left(\mathrm{m}^{3}\right)$ and dry matter $(\mathrm{kg})$ in families of the Pinus tecunumanii Mountain Pine Ridge provenance grown at Planaltina, Federal District, Brazil, with 12 years of age: Means were compared with all plot trees (6 individuals) and with a sample of best plot trees (3 individuals).

\begin{tabular}{c|l|l|c|c|c|c|c|c}
\hline \multicolumn{3}{c|}{ Densidade } & \multicolumn{3}{c|}{ Volume } & \multicolumn{3}{c}{ Matéria seca } \\
\hline Família & 3 indiv. & 6 indiv. & Família & 3 indiv. & 6 indiv. & Família & 3 indiv. & 6 indiv. \\
\hline 482 & $0,45 \mathrm{a}$ & $0,44 \mathrm{a}$ & 452 & 0,22 & $0,19 \mathrm{a}$ & 452 & $98 \mathrm{a}$ & $83 \mathrm{a}$ \\
452 & $0,44 \mathrm{ab}$ & $0,45 \mathrm{a}$ & 461 & 0,22 & $0,17 \mathrm{ab}$ & 461 & $91 \mathrm{ab}$ & $73 \mathrm{ab}$ \\
487 & $0,43 \mathrm{abc}$ & $0,43 \mathrm{ab}$ & 482 & 0,20 & $0,16 \mathrm{ab}$ & 482 & $91 \mathrm{ab}$ & $73 \mathrm{ab}$ \\
448 & $0,42 \mathrm{bcd}$ & $0,42 \mathrm{bc}$ & 444 & 0,19 & $0,14 \mathrm{bc}$ & 444 & $77 \mathrm{bc}$ & $58 \mathrm{bc}$ \\
461 & $0,42 \mathrm{~cd}$ & $0,42 \mathrm{bc}$ & 448 & 0,18 & $0,15 \mathrm{bc}$ & 448 & $74 \mathrm{bc}$ & $62 \mathrm{bc}$ \\
444 & $0,41 \mathrm{~cd}$ & $0,40 \mathrm{c}$ & 487 & 0,17 & $0,14 \mathrm{bc}$ & 487 & $73 \mathrm{c}$ & $61 \mathrm{bc}$ \\
462 & $0,40 \mathrm{~d}$ & $0,41 \mathrm{bc}$ & 462 & 0,16 & $0,12 \mathrm{c}$ & 462 & $64 \mathrm{c}$ & $51 \mathrm{c}$ \\
\hline Médias & 0,42 & 0,42 & \multicolumn{7}{c}{0,19} & 0,15 & & 81,1 & 65,7 \\
\hline
\end{tabular}

Médias seguidas por letras distintas entre as linhas diferem entre si ao nível de 5\%.

Nas Idades 1 e 2, a densidade básica média da madeira foi de respectivamente 0,425 e $0,456 \mathrm{~g} / \mathrm{cm}^{3}$ (Tabela 3); aumentando em média $0,031 \mathrm{~g} / \mathrm{cm}^{3}$ em 5 anos. Já é fato comum o aumento da densidade básica da madeira com a idade, como foi demonstrado nos trabalhos de Rocha et al. (1983) e Higa et al. (1973).

$\mathrm{Na}$ idade 1, a faixa de variação da densidade básica entre indivíduos foi de $0,35 \mathrm{a} 0,49 \mathrm{~g} / \mathrm{cm}^{3}$, menor do que na idade 2 , cuja variação foi de 0,37 a $0,60 \mathrm{~g} / \mathrm{cm}^{3}$. As diferenças entre as médias de famílias permaneceram constantes da primeira para a segunda idade, de 0,40 a $0,45 \mathrm{~g} / \mathrm{cm}^{3}$ na idade 1 e de 0,43 a 0,48 $\mathrm{g} / \mathrm{cm}^{3}$ na idade 2 (Tabela 3). Embora a correlação entre a densidade básica da madeira nas duas idades tenha sido positiva e significativa, $(\mathrm{r}=0,24, \mathrm{P}<0.001)$, alguns indivíduos não seguiram essa tendência, isto é, a densidade permaneceu constante ou diminuiu com o passar dos anos (Figura 1). Essa inconstância na tendência de variação da densidade com a idade, por parte de uma parcela dos indivíduos, explicam o fato de que os resultados das análises da variância para essa característica tenham sido diferentes para as duas idades. 
TABELA 3: Teste de Waller-Duncan para as características de Densidade básica $\left(\mathrm{g} / \mathrm{cm}^{3}\right)$, Volume $\left(\mathrm{m}^{3}\right)$ e Matéria Seca (kg), em famílias da procedência de Mountain Pine Ridge, de Pinus tecunumanii, coletados em Planaltina - DF: comparação entre as médias aos 12 e 17 anos de idade.

TABLE 3: Waller-Duncan test for the characteristics wood of density $\left(\mathrm{g} / \mathrm{cm}^{3}\right)$, Volume $\left(\mathrm{m}^{3}\right)$ and dry matter in families of the Pinus tecunumanii Mountain Pine Ridge provenance grown at Planaltina, Federal District, Brazil: Means comparison at 12 and 17 years of age.

\begin{tabular}{c|ll|l|lll|c|c|c}
\hline \multicolumn{3}{c|}{ Densidade } & \multicolumn{3}{c|}{ Volume } & \multicolumn{3}{c}{ Matéria seca } \\
\hline Família & Média12 & Média17 & Família & Média12 & Média17 & Família & Média12 & Média17 \\
\hline 482 & $0,45 \mathrm{a}$ & 0,48 & 452 & $0,22 \mathrm{a}$ & $0,44 \mathrm{ab}$ & 452 & $98 \mathrm{a}$ & $157 \mathrm{a}$ \\
452 & $0,44 \mathrm{ab}$ & 0,47 & 461 & $0,22 \mathrm{a}$ & $0,48 \mathrm{a}$ & 461 & $91 \mathrm{ab}$ & $156 \mathrm{a}$ \\
487 & $0,43 \mathrm{abc}$ & 0,47 & 482 & $0,20 \mathrm{ab}$ & $0,38 \mathrm{c}$ & 482 & $91 \mathrm{ab}$ & $125 \mathrm{~b}$ \\
448 & $0,42 \mathrm{bcd}$ & 0,46 & 444 & $0,19 \mathrm{abc}$ & $0,39 \mathrm{bc}$ & 444 & $77 \mathrm{bc}$ & $127 \mathrm{~b}$ \\
461 & $0,42 \mathrm{~cd}$ & 0,45 & 448 & $0,18 \mathrm{bc}$ & $0,32 \mathrm{~cd}$ & 448 & $74 \mathrm{bc}$ & $106 \mathrm{bc}$ \\
444 & $0,41 \mathrm{~cd}$ & 0,44 & 487 & $0,17 \mathrm{bc}$ & $0,29 \mathrm{~d}$ & 487 & $73 \mathrm{c}$ & $101 \mathrm{bc}$ \\
462 & $0,40 \mathrm{~d}$ & 0,43 & 462 & $0,16 \mathrm{c}$ & $0,28 \mathrm{~d}$ & 462 & $64 \mathrm{c}$ & $88 \mathrm{c}$ \\
\hline Médias & 0,43 & 0,46 & \multicolumn{3}{c}{0,19} & 0,36 & & 81,1 & 168,3 \\
\hline
\end{tabular}

Médias seguidas por letras distintas entre as linhas diferem entre si ao nível de 5\%.

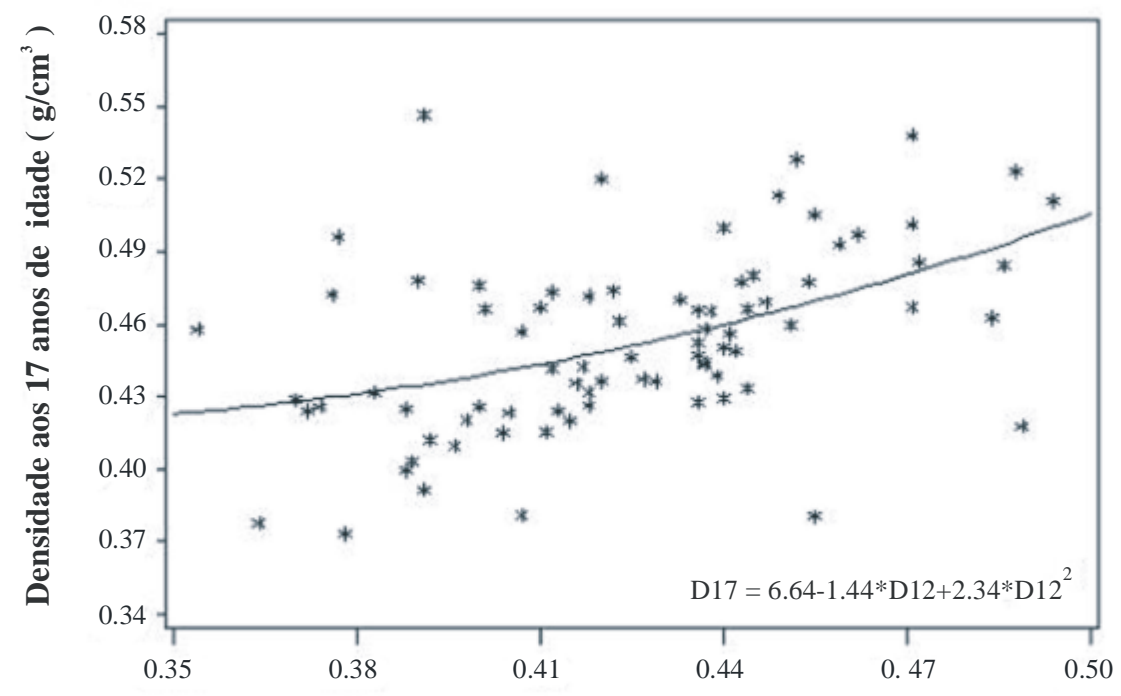

Densidade aos 12 anos de idade ( $\left.\mathrm{g} / \mathrm{cm}^{3}\right)$

FIGURA 1: Relação entre a densidade básica da madeira medida aos 12 e aos 17 anos de idade em Pinus tecunumanii testados em Planaltina-DF.

FIGURE 1: Relationship between wood basic density at 12 and 17 years of age of Pinus tecunumanii tested at Planaltina, DF.

$\mathrm{Na}$ idade 1 , as diferenças entre as densidades médias de famílias foram altamente significativas ( $\mathrm{F}$ $=4,14, \mathrm{p}<0,001$ ), porém, o mesmo não aconteceu na idade 2, embora a ordem das famílias com maior ou menor densidade permanecesse inalterada nas duas idades (Tabela 3). Essa mudança de comportamento de uma idade para a outra pode ser explicada pelas variações entre as amostragens feitas nas duas idades, já que os cálculos foram feitos em baguetas coletadas ao nível do DAP, porém, em idades diferentes, constituindo-se assim em unidades distintas. A adição de anéis de crescimento ao longo dos 5 anos, com perímetro maior dos anteriores e variando de indivíduo para individuo, especialmente quando estes estão submetidos a uma maior competição, pode subestimar ou superestimar as densidades encontradas, pois ela não é ponderada em relação aos diâmetros dos anéis. A competição entre indivíduos de um mesmo talhão, aumenta com a idade, 
favorecendo os indivíduos dominantes o que pode refletir positiva ou negativamente na densidade da madeira de cada indivíduo. A não-detecção de diferenças significativas na idade 2, demonstra que, com a idade, os fatores ambientais e de competição podem mascarar diferenças genéticas para a característica densidade. Dessa maneira, as seleções tanto para características de crescimento como de densidade devem ser feitas em idades mais jovens, em que a competição entre indivíduos ainda não é tão acentuada.

A herdabilidade da densidade básica calculada na idade 1, em nível individual, para família e dentro de família, para a amostragem 1 , foi de $0,43,0,81$ e 0,33 e para amostragem 2 , foi de $0,47,0,63,0,37$ (Tabela 4). A herdabilidade individual e a herdabilidade dentro de família foi menor quando apenas foram considerados os três indivíduos dentro da parcela, porém o inverso aconteceu para a herdabilidade em nível de família. Esse resultado se deve à diminuição do coeficiente de variação entre indivíduos dentro de cada família. O coeficiente de variação foi de $8,71 \%$ quando todos os indivíduos foram mensurados e a redução de $50 \%$ no número de indivíduos amostrados acarretou uma diminuição no coeficiente de variação para 6,54 \%.

TABELA 4: Estimativa das herdabilidades para densidade básica, volume e índice de matéria seca, em nível individual $\left(\mathrm{h}^{2} \mathrm{i}\right)$, para família $\left(\mathrm{h}^{2} \mathrm{f}\right)$ e dentro de família $\left(\mathrm{h}^{2} \mathrm{w}\right)$ em Pinus tecunumanii testado em Planaltina-DF, aos 12 anos de idade, utilizando amostragem com todos os indivíduos da parcela e com apenas três indivíduos superiores da parcela.

TABLE 4: Heritability estimates of wood density, volume and dry matter index at individual $\left(h^{2} \mathrm{i}\right)$, family $\left(\mathrm{h}^{2} \mathrm{f}\right)$ and within family $\left(\mathrm{h}^{2} \mathrm{w}\right)$ levels of Pinus tecunumanii grown at Planaltina, Federal District, Brazil with 12 years of age, where the sampling was done with all plot trees and with the 3 superior trees in the plot.

\begin{tabular}{l|c|c|c|c|c|c}
\hline \multirow{2}{*}{ Características } & \multicolumn{3}{|c|}{ Todos os indivíduos da parcela } & \multicolumn{3}{c}{ Três maiores indivíduos/parcela } \\
\cline { 2 - 7 } & $\mathrm{h}^{2} \mathrm{i}$ & $\mathrm{h}^{2} \mathrm{f}$ & $\mathrm{h}^{2} \mathrm{w}$ & $\mathrm{h}^{2} \mathrm{i}$ & $\mathrm{h}^{2} \mathrm{f}$ & $\mathrm{h}^{2} \mathrm{w}$ \\
\hline Densidade básica & 0,43 & 0,81 & 0,33 & 0,47 & 0,63 & 0,37 \\
Volume & 0,33 & 0,73 & 0,24 & 0,43 & 0,62 & 0,33 \\
Matéria Seca & 0,39 & 0,77 & 0,29 & 0,49 & 0,64 & 0,39 \\
\hline
\end{tabular}

Diferenças significativas não foram verificadas entre as médias da densidade básica da madeira na idade 2. O cálculo da herdabilidade foi feito apenas para indivíduos, já que este foi de 0,14 , inferior ao encontrado para a idade 1, como era esperado. Dessa maneira, os ganhos genéticos serão superiores se a seleção for feita na idade 1 , como já foi dito anteriormente.

A ordem das médias de família das densidades básica permaneceu constante, quando consideradas os dois tipos de amostragem e as duas idades (Tabelas 2 e 3 ).

As médias de volume na idade 1, considerando-se as amostragens 1 e 2, foram respectivamente 0,190 $\mathrm{m}^{3}$, variando de 0,160 a $0,220 \mathrm{~m}^{3}$ e de $0,154 \mathrm{~m}^{3}$ variando de 0,120 a $0,190 \mathrm{~m}^{3}$ (Tabela 2). O coeficiente de variação foi maior na amostragem $1(\mathrm{cv}=40,3 \%)$ do que na amostragem $2(\mathrm{cv}=29.9 \%)$. Houve diferenças significativas em volume entre média de famílias aos 12 anos $(F=3,64, p<0,004)$. Na idade 2 (17 anos), não foi feita análise de variância nem cálculo de herdabilidades para volume, pela eliminação de indivíduos. As herdabilidades para volume, considerando as duas amostragens, apresentaram comportamento idêntico ao da densidade básica; com diminuição apenas da herdabilidade em nível de família da amostragem 1 para a 2 (Tabela 4). As herdabilidades para volume e matéria seca não foram calculadas, pois quando os maiores indivíduos dentro de cada parcela foram selecionados, isso já representou ganho genético em volume.

$\mathrm{Na}$ idade 2, a média de volume foi de $0,268 \mathrm{~m}^{3}$ (Tabela 5). Para massa seca, houve diferenças significativas entre médias de famílias, tanto aos $12(\mathrm{~F}=4,44, \mathrm{p}<0,001)$ como aos 17 anos $(\mathrm{F}=6,65, \mathrm{p}<$ 0,001). A média de massa seca variou de $81 \mathrm{~kg}$ na idade de 12 anos para $168 \mathrm{~kg}$ na idade de 17 anos, influenciada mais pelo volume do que pela densidade, sobretudo na última idade. 
TABELA 5: Médias de volume $\left(\mathrm{m}^{3}\right)$ e de matéria seca $(\mathrm{kg})$ de famílias da procedência de Mountain Pine Ridge de Pinus tecunumanii, coletados em Planaltina, DF, aos 17 anos de idade.

TABLE 5: Family means of volume $\left(\mathrm{m}^{3}\right)$ and dry matter $(\mathrm{kg})$ of the Pinus tecunumanii Mountain Pine Ridge provenance, grown at Planaltina, Federal District, Brazil, with 17 years of age.

\begin{tabular}{c|c|c|c}
\hline Família & Volume & Família & Matéria seca \\
\hline 461 & $0,35 \mathrm{a}$ & 461 & $156 \mathrm{a}$ \\
452 & $0,34 \mathrm{ab}$ & 452 & $157 \mathrm{a}$ \\
444 & $0,28 \mathrm{bc}$ & 482 & $125 \mathrm{~b}$ \\
482 & $0,26 \mathrm{~cd}$ & 444 & $127 \mathrm{~b}$ \\
448 & $0,22 \mathrm{de}$ & 448 & $106 \mathrm{bc}$ \\
487 & $0,21 \mathrm{de}$ & 487 & $101 \mathrm{bc}$ \\
462 & $0,20 \mathrm{e}$ & 462 & $88 \mathrm{c}$ \\
\hline Médias & 0,27 & & 123,57 \\
\hline
\end{tabular}

Médias seguidas por letras distintas entre as linhas diferem entre si ao nível de 5\%.

\section{CONCLUSÕES}

Não houve diferença entre os dois tipos de amostragem realizadas e, conseqüentemente, o uso de indivíduos superiores para a determinação da densidade básica da madeira é perfeitamente aceitável.

As seleções para densidade básica devem ser feitas em povoamento com idade por volta dos 12 anos de idade, quando a competição entre os indivíduos ainda não atingiu níveis elevados.

Diferenças significativas foram encontradas entre médias de famílias aos 12 e aos 17 anos de idade, exceto para a característica densidade básica.

Houve correlação positiva entre a densidade básica da madeira medida aos 12 e 17 anos de idade.

Ganhos genéticos em volume, densidade básica e matéria seca serão possíveis na seleção de indivíduos ou famílias dentro da procedência de MPR, Belize, na região do Cerrado.

\section{REFERÊNCIAS BIBLIOGRÁFICAS}

BIRKS, J.S.; BARNES, R.D. Provenance variation in Pinus caribaea, Pinus oocarpa and Pinus patula ssp. tecunumanii. Oxford: University of Oxford,Oxford Forestry Institute, Department of Plant Sciences, 1990. 40 p. (Tropical Forestry Papers 21).

BARRICHELO, L.E.G.; KAGEYAMA, P.Y.; SPELTZ, R.M.; BONISH, H.J.; BRITO, J.O.; FERREIRA,M. Estudo de procedências de Pinus taeda visando seu aproveitamento industrial. IPEF, Piracicaba, v.15, p. 1-14, 1977.

BRASIL, M.A.M.; NICOLLELO, N.; VEIGA, R. A. A. Variação da densidade básica da madeira de Pinus oocarpa Schiede em diversas idades na região de Agudos - SP. Revista. Floresta, Curitiba-PR, v.11, n.1, p. 33-39, 1980.

EGUILUZ-PIEDRA, T.; PERRY, J.P. Jr. Pinus tecunumanii: uma espécie nueva de Guatemala. Ciência Florestal, v.8, n.41, p.3-22, 1983.

FERREIRA, M. Estudo da variação da densidade básica da madeira de Eucalyptus alba Reiwn e Eucalyptus saligna SMITH. IPEF, Piracicaba, v.1, p.83-96, 1970.

GIBSON, G.L. Genotype: environment interaction in Pinus caribaea. Oxford, Commonwealth Forestry Institute, University of Oxford. 1982. $112 \mathrm{p}$.

HIGA, A.R.; KAGEYAMA, P.Y.; FERREIRA, M. Variação da densidade básica da madeira de Pinus elliottii var. elliottii e Pius taeda. IPEF, Piracicaba, v. 7, p.79-91, 1973.

LADRACH, W. E. Comparaciones entre procedencias de siete coniferas en la Zona Andina al finalizar los ocho años. Colombia : Smurfit Cartón de Colombia, 1986. 8 p. (Informe de Investigación, 105).

LIMA, R.T.; JETT, J.B.; DVORAK, W.S. Family stability of wood specific gravity in Pinus tecunumanii established on three sites in South America. New Forest, v.3, n.316, p.311, 1990.

MOURA, V.P.G.; SANTIAGO, J. Densidade básica da madeira de espécies de Pinus tropicais determinada através de 
métodos não-destrutivos. Planaltina: EMBRAPA-CPAC, 1991. 14p. (Boletim de Pesquisa, 33).

MOURA, V.P.G.; OLIVEIRA, J. B., REZEK JUNIOR, J. Variabilidade e ganho genético em progênies de meioirmãos de Pinus patula ssp. tecunumanii, em Planaltina-DF. In.: SIMPÓSIO INTERNACIONAL SOBRE ECOSSISTEMAS FLORESTAIS, 4.. 1996, Belo Horizonte. Anais... Belo Horizonte: Sociedade Brasileira para a Valorização do Meio Ambiente - Biosfera, 1996. p.230-233.

ROCHA, M.G.B.; BRUNE, A.; LUCIA, R.M.D.; OLIVEIRA L.M. Variação da densidade básica e correlações entre caracteres progênies jovens de Eucalyptus grandis Hill ex Maiden, em duas etapas de crescimento. Revista Árvore, Viçosa, v.7, n. 2, p.154-164, 1983.

ROSADO, S.C. da S.; BRUNE, A. Crescimento de árvores: estimativa de correlações entre idades diferentes e sua influência da densidade básica da madeira em Eucalyptus ssp. Revista Árvore, Viçosa, v.7, n.1, p. 11-22, 1983.

SAS/STAT. User's Guide: version 6. 4th ed. Cary, NC. USA, 1990. v.2.

SMITH, D.M. Maximum moisture content method for determining specific gravity of small wood samples. Madison, 1954. 8 p. (USDA, FPL, 2014).

SQUILLACE, A.E. Average genetic correlations among off-sprin from open-pollinated forest trees. Silvae Genética, v. 23, p.149-156, 1974.

VASQUEZ, J.; DVORAK, W.S. Trend in variances and heritabilities with stand development of tropical pines. Canadian Journal of Forest Research, v. 26, p. 1473-1480, 1996.

WRIGHT, J.A.; GIBSON.G.L.; BARNES, R.D. Variation of stem volume and wood density of provenances of Pinus oocarpa and Pinus patula ssp. tecunumanii at Agudos, São Paulo, Brazil. IPEF, Piracicaba, v.32, p.21-23, 1986a.

WRIGHT, J.A.; GIBSON, G.L.; BARNES, R.D. Provenance variation in stem volume and wood density of Pinus caribaea, P. oocarpa, and P. patula ssp. tecunumanii in Zambia. Commonwealth Forestry Review, v. 65, n.1, p.3341, $1986 \mathrm{~b}$.

WRIGHT, J.A. Results of micro pulping wood samples of Pinus caribaea, P. elliottii, P. oocarpa and P. patula ssp. tecunumanii in the Eastern Transvaal and Zululand. In: SIMPOSIO SOBRE SILVICULTURA Y MEJORAMIENTO DE ESPECIES FORESTALES, 1987, Buenos Aires. CIEF ... Buenos Aires, 1987. p. 247-256. v.4.

WRIGHT, J. A. Variation in wood properties of Pinus oocarpa and Pinus patula ssp. tecunumanii at six sites. Silvae Genetica, v.39, n.1, p. 1-5, 1990.

WRIGHT, J.A.; GIBSON.G.L.; BARNES, R.D. Variation in volume and wood density of eight provenances of Pinus oocarpa and Pinus patula ssp. tecunumanii in Conocotto, São Paulo, Brazil. IPEF, Piracicaba, v. 41, p.55-57, 1990. 